\title{
Hindgut neuroendocrine neoplasms - characteristics and prognosis
}

Paweł Gut, Joanna Waligórska-Stachura, Agata Czarnywojtek, Nadia Sawicka-Gutaj, Maciej Bączyk, Katarzyna Ziemnicka, Kosma Woliński, Ariadna Zybek, Jakub Fischbach, Marek Ruchała

Department of Endocrinology, Poznan University of Medical Sciences, Poznan, Poland

Submitted: 8 May 2016

Accepted: 18 October 2016

Arch Med Sci 2017; 13, 6: 1427-1432

DOI: 10.5114 /aoms.2017.64979

Copyright (c) 2017 Termedia \& Banach

\section{Abstract}

Introduction: The aim of the study was to analyze the clinicopathologic characteristics and prognostic factors of hindgut-rectal neuroendocrine neoplasms.

Material and methods: The study included 38 patients with rectal neuroendocrine tumors who were treated at the Department of Endocrinology, Metabolism and Internal Diseases, Poznan University of Medical Sciences, Poznan, Poland from February 2010 to December 2015. The clinicopathological data were retrospectively reviewed, extracted, analyzed, and patients were followed up to determine their survival status. Follow-up data were available for all 38 patients. Uni- and multivariate Cox regression analyses were performed to determine the prognostic factors significantly associated with overall survival.

Results: The tumors occurred mostly in the middle and lower rectum, and the most typical symptoms experienced by patients were hematochezia and diarrhea. The median distance between the tumors and the anal edges was $4.7 \pm 1.3 \mathrm{~cm}$, and the median diameter of the tumors was $0.9 \pm 1.2 \mathrm{~cm}$. The major pathological types were neuroendocrine neoplasm G1 in 31 patients, and neuroendocrine neoplasm G2 in 7 patients. Tumor-node-metastasis (TNM) stages I, II, III and IV tumors accounted for $76.3 \%$ (29/38), 5.3\% $(2 / 38), 7.9 \%(3 / 38)$ and $10.5 \%(4 / 38)$ of patients, respectively. The main treatment method was transanal extended excision or endoscopic resection. The 1-, 3- and 5-year survival rates of the whole group of patients were $100 \%, 83.7 \%$, and $75.3 \%$, respectively.

Conclusions: Univariate analysis showed that age $(p=0.022)$, tumor diameter $(p<0.001)$, histological type $(p<0.001)$, and TNM stage $(p<0.001)$ were all prognostic factors.

Key words: prognostic factors, clinical characteristics, hindgut tumors, rectal neuroendocrine tumors.

\section{Introduction}

The rectum is the second most frequent site $(27.4 \%)$ of gastrointestinal neuroendocrine neoplasia (NEN) occurrence and is surpassed in prevalence only by the small intestine. Approximately $50 \%$ of patients are asymptomatic at presentation. Rectal pain, pruritus, hematochezia, weight loss, and constipation occur as components of late presentation. Despite the fact that rectal NENs can secrete hormonal products directly into the systemic circulation, the carcinoid syndrome occurs very rarely.

\author{
Corresponding author: \\ Pawet Gut MD, PhD \\ Department of Endocrinology \\ Poznan University \\ of Medical Sciences \\ 49 Przybyszewskiego St \\ 60-355 Poznan, Poland \\ Phone: +48 618691322 \\ E-mail: gutpj@poczta.onet.pl
}


Rectal NENs, however, comprise only $1-2 \%$ of all rectal tumors und exhibit the most benign clinical profile of NENs, possibly reflecting their early diagnosis by endoscopic examination. Rectal neuroendocrine tumors are rare, with an incidence of about 3.08 per 100000 persons per year [1-3]. They are considered to be a type of tumor with indolent biological behavior and a relatively favorable prognosis [4]. Modlin et al. [1] reported a 5-year survival rate of $88.3 \%$. Bernick et al. [5] stated that the 3-year survival rate in a group of patients with colorectal neuroendocrine tumors was only $13 \%$. This indicates significant differences between reported results. Such large differences in the results were associated with a too small study group and the wide diversity of histological grade in Bernick's design. An analysis of the clinicopathologic characteristics of a group of patients with pathologically confirmed diagnoses of rectal neuroendocrine tumors showed that these tumors mostly occur in the middle and lower rectum. Overall, rectal NENs fall into two groups, small solitary tumors measuring less than $1 \mathrm{~cm}$ and larger lesions with the possibility of metastases. In general, rectal NENs metastasize in $2 \%$ of tumors less than $1 \mathrm{~cm}, 10-12 \%$ of tumors less than $2 \mathrm{~cm}$ and $65-75 \%$ of tumors more than $2 \mathrm{~cm}$ [6-8]. The most common tumor-node-metastasis stage found was stage I, and lymph node or distant metastases were rarely seen. The major pathological type is a neuroendocrine neoplasm in histological grades $G 1$ and G2. Transanal extended excisions generally produced satisfactory curative effects, and the 5-year survival rate is usually as high as $75.3 \%$ [9-11]. This study was undertaken to analyze the clinicopathologic characteristics of rectal neuroendocrine tumors in a group of patients seen at our department between February 2010 and December 2015, and to identify the prognostic factors for their survival.

\section{Material and methods}

The study was a retrospective analysis of $38 \mathrm{pa}$ tients with rectal neuroendocrine tumors in whom a definite pathological diagnosis had been made at the Pathology Department, Poznan University of Medical Sciences, Poznan, Poland. The patients included 21 males and 17 females, with a median age of 51.7 years (range: $31-67$ years). The tumors were staged via the TNM staging standard for rectal neuroendocrine tumors, which was updated by the European Neuroendocrine Tumor Society in 2007 [12, 13].

\section{Statistical analysis}

The Kaplan-Meier method was applied for the survival analysis. A log-rank test was used for a univariate analysis of prognostic factors, and a Cox proportional hazard model was used for a multivariate analysis. A $p$-value $<0.05$ was considered statistically significant.

\section{Results}

All patients underwent a colonoscopy. The most common symptoms in the 38 patients evaluated were hematochezia in $31.5 \%(12 / 38)$ and diarrhea in $26.3 \%(10 / 38)$. None of the patients exhibited hormonal symptoms of the tumor activity. The median distance between the tumors and anal edges was $4.7 \pm 1.3 \mathrm{~cm}$. Patients with a distance between the tumor and the anal edge $\leq 8 \mathrm{~cm}$ accounted for $94.7 \%$ (36/38) of the group. All patients were diagnosed pathologically as neuroendocrine tumors by biopsy and histopathology after surgery according to the World Health Organization (WHO) 2010 diagnostic criteria for gastrointestinal neuroendocrine tumors [14-16]. The pathological types included 31 cases of neuroendocrine tumors in stage $\mathrm{G} 1(\mathrm{Ki}-67<2 \%)$ and 7 cases of neuroendocrine tumors in stage G2 (Ki-67, 2-20\%). The median diameter of the tumors was $0.9 \pm 1.2 \mathrm{~cm}$. In 21 patients, the diameters were 0.1 to $0.8 \mathrm{~cm}$; in 13 they were 0.9 to $1.9 \mathrm{~cm}$; and in 4 they were $\geq 2 \mathrm{~cm}$. Immunohistochemical staining showed that the tumors were positive for chromogranin A (CgA) and synaptophysin (Syn). All 38 cases had a CT scan of the abdomen and pelvis to determine whether there were metastases to the lymph nodes or distant organs. The distant metastasis rate was $10.5 \%(4 / 38)$ at the time of diagnosis. All patients were staged according to the TNM staging system for rectal neuroendocrine tumors (Tables I and II) [17, 18]. Stages I, II, III and IV tumors accounted for $76.3 \%$ (29/38), 5.3\% (2/38), $7.9 \%$ (3/38), and $10.5 \%$ (4/38) of patients, respectively. Surgical treatment was undertaken in all of the patients, including transanal extended excision and endoscopic resection [19, 20]. Four patients received locoregional therapy because they were initially diagnosed with stage IV disease with liver metastasis. The median survival time in the patients studied was not reached. The 1-, 3- and 5-year survival rates were 100\%, 83.7\% and $75.3 \%$, respectively. Four patients demonstrated recurrence and metastases after radical resection, and the mean time for recurrence of metastasis was 14 months. Log-rank analysis of prognostic factors showed that there was a statistically significant difference in the 5 -year survival rate between patients $\geq 65$ years of age and patients $<65$ years of age $(p=0.022)$. Subgroup analysis stratified by TNM stage and tumor type showed that the 5 -year survival rate in patients aged $\geq 65$ years was lower in those with neuroendocrine tumor G2 at TNM stage III/IV. In terms 
of tumor diameters, the patients were classified into 3 subgroups: those with tumor diameters $<1.0 \mathrm{~cm}$, between 1.0 and $2.0 \mathrm{~cm}$, and $>2.0 \mathrm{~cm}$. There was a statistically significant difference in the overall survival between these subgroups $(p<0.001)$, and also between the pathological types of neuroendocrine tumors $G 1$ and $G 2$ $(p<0.001)$. Clinicopathologic characteristics of rectal-hindgut neuroendocrine tumor patients are presented in Table III.

\section{Discussion}

Rectal neuroendocrine tumors account for $1 \%$ to $2 \%$ of all rectal tumors, and occur mostly in the 60-70-year-old age group [21-23]. Data on 1481 cases of rectal neuroendocrine tumors occurring over a period of 30 years in the United States showed that males accounted for $51.7 \%$ of the overall incidence [24-26]. In the present study, the most common age of onset was 31-67 years (median of 51.7 years), with males accounting for $55.2 \%$ of the overall incidence. This can be compared with other data, which show a trend towards a younger age of onset and a higher incidence in males. Nearly $50 \%$ of our patients with rectal neuroendocrine tumors showed no obvious symptoms at the time the diagnosis was confirmed. Rather, the tumors were generally found by conventional colonoscopy. In patients with symptoms, rectal bleeding, pain, and constipation were noted most commonly [27-29]. Rectal neuroendocrine tumors can arise in the entire rectum. In this study, the median distance between the tumors and the anal edges was $4.7 \mathrm{~cm}$, and patients with a distance of $\leq 8 \mathrm{~cm}$ between the tumor and the anal edge accounted for $94.7 \%$ of all cases, indicating that the tumors mostly arise in the middle and lower rectum. The CgA and synaptophysin are commonly used as biomarkers to detect neuroendocrine tumors. In the group of patients we studied, positive immunohistochemical staining rates for these markers were $100 \%$ and $76.5 \%$. This indicates that CgA staining is more sensitive
Table I. Proposed tumor-node-metastasis classification for neuroendocrine tumors of the rectum (European Neuroendocrine Tumor Society 2007)

\begin{tabular}{|l|}
\hline TNM classification \\
\hline T; primary tumor: \\
\hline T0; no evidence of primary tumor \\
\hline T1; tumor invades the mucosa or submucosa and \\
size $\leq 1$ cm \\
\hline T1a; size $<1$ cm \\
\hline T1b; size $1-2$ cm \\
\hline T2; tumor invades the muscularis propria at size $>2 \mathrm{~cm}$ \\
\hline T3; tumor invades subserosa/pericolic/perirectal fat \\
\hline T4; tumor directly invades other organs/structures \\
and/or perforates the visceral peritoneum \\
\hline $\mathrm{N} ;$ regional lymph nodes: \\
\hline $\mathrm{Nx}$; regional lymph nodes cannot be assessed \\
\hline $\mathrm{N} 0 ;$ no regional lymph node metastasis \\
\hline $\mathrm{N} 1 ;$ regional lymph node metastasis \\
\hline $\mathrm{M}$; distant metastasis: \\
\hline M tumor-node-metastasis.
\end{tabular}

for the diagnosis of rectal neuroendocrine tumors than synaptophysin. It has been reported in the literature that the most common sites of metastases of rectal neuroendocrine tumors are lymph nodes and the liver, and only rarely the lungs [3033]. In the present study 4 patients with $G 2$ neuroendocrine tumors that led to surgery presented metastasis to a lymph node or the liver. The lymph node metastases involved nodes adjacent to the iliac arteriovenous, retroperitoneal and inguinal lymph nodes $[34,35]$. In our study, the overall

Table II. Disease staging for neuroendocrine tumors of the colon and rectum

\begin{tabular}{|c|c|c|c|}
\hline Disease stage & $\mathrm{T}$; Primary tumor & N; Regional nodes & M; Distant metastasis \\
\hline I A & $\mathrm{T} 1 \mathrm{a}$ & NO & MO \\
\hline I B & $\mathrm{T} 1 \mathrm{~b}$ & NO & MO \\
\hline II A & $\mathrm{T} 2$ & NO & MO \\
\hline II B & T3 & NO & MO \\
\hline III A & T4 & NO & MO \\
\hline III B & Any $T$ & N1 & MO \\
\hline IV & Any $\mathrm{T}$ & Any $N$ & M1 \\
\hline
\end{tabular}


Table III. Clinicopathologic characteristics of rectal neuroendocrine tumor patients

\begin{tabular}{|c|c|c|c|c|c|c|c|c|c|c|c|c|}
\hline No. & Patient & $\begin{array}{c}\text { Age } \\
\text { [years] }\end{array}$ & $\begin{array}{l}\text { Sex } \\
(F / M)\end{array}$ & $\begin{array}{l}\text { Grading } \\
\text { (G1/G2) }\end{array}$ & $\begin{array}{c}\mathrm{Ki}-67 \\
(\%)\end{array}$ & $\mathrm{CgA}$ & Syn. & TNM & $\begin{array}{c}\text { Tumor } \\
\text { size }[\mathrm{cm}]\end{array}$ & $\begin{array}{l}\text { Distance from } \\
\text { anal edge }[\mathrm{cm}]\end{array}$ & $\begin{array}{l}\text { Feb. } \\
2010\end{array}$ & $\begin{array}{l}\text { Dec. } \\
2015\end{array}$ \\
\hline 1 & A.K. & 31 & $M$ & G1 & 1 & +++ & +++ & 1 & 1.4 & 8.0 & SD & PD \\
\hline 2 & B.T. & 43 & M & G1 & 2 & +++ & + & 1 & 0.9 & 6.5 & SD & SD \\
\hline 3 & C.K. & 51 & $\mathrm{~F}$ & G1 & 2 & ++ & - & 1 & 0.2 & 7.5 & SD & SD \\
\hline 4 & T.P. & 66 & M & $\mathrm{G} 2$ & 5 & ++ & - & IV & 2.8 & 4.5 & PD & Died \\
\hline 5 & W.M. & 35 & M & G1 & 1 & +++ & ++ & I & 0.1 & 2.5 & SD & SD \\
\hline 6 & E.T. & 39 & $\mathrm{~F}$ & G1 & 2 & +++ & ++ & 1 & 0.4 & 2.0 & SD & SD \\
\hline 7 & P.G. & 42 & $M$ & G1 & 1 & ++ & +++ & 1 & 0.7 & 3.5 & SD & SD \\
\hline 8 & M.G. & 56 & $\mathrm{~F}$ & $\mathrm{G} 2$ & 10 & + & - & IV & 2.9 & 2.0 & PD & Died \\
\hline 9 & E.G. & 55 & $M$ & G1 & 2 & ++ & ++ & 1 & 0.6 & 2.5 & SD & SD \\
\hline 10 & M.R. & 47 & $\mathrm{~F}$ & G1 & 1 & +++ & +++ & I & 1.3 & 7.5 & SD & SD \\
\hline 11 & J.K. & 46 & $M$ & G1 & 2 & +++ & ++ & 1 & 0.3 & 5.5 & SD & SD \\
\hline 12 & O.K. & 66 & $M$ & G1 & 2 & ++ & ++ & II & 1.5 & 4.5 & SD & PD \\
\hline 13 & P.K. & 32 & $\mathrm{~F}$ & G1 & 2 & ++ & ++ & I & 0.4 & 3.5 & SD & SD \\
\hline 14 & R.T. & 34 & $M$ & G1 & 1 & +++ & ++ & 1 & 0.2 & 6.5 & SD & SD \\
\hline 15 & O.G. & 66 & $M$ & $\mathrm{G} 2$ & 10 & + & - & IV & 2.4 & 5.5 & PD & Died \\
\hline 16 & J.L. & 62 & $\mathrm{~F}$ & G1 & 2 & +++ & ++ & 1 & 0.4 & 2.5 & SD & SD \\
\hline 17 & W.K. & 41 & $\mathrm{~F}$ & G1 & 1 & +++ & ++ & I & 0.8 & 3.5 & SD & SD \\
\hline 18 & P.K. & 57 & $M$ & G1 & 2 & ++ & ++ & 1 & 0.2 & 4.5 & SD & SD \\
\hline 19 & H.S. & 65 & $\mathrm{~F}$ & $\mathrm{G} 2$ & 5 & ++ & - & III & 1.9 & 4.0 & PD & Died \\
\hline 20 & K.B. & 36 & $M$ & G1 & 2 & +++ & ++ & I & 0.8 & 3.0 & SD & SD \\
\hline 21 & E.O. & 47 & $\mathrm{~F}$ & G1 & 1 & +++ & ++ & I & 0.5 & 2.0 & SD & SD \\
\hline 22 & G.F. & 52 & $M$ & G1 & 2 & ++ & ++ & I & 0.7 & 4.5 & SD & SD \\
\hline 23 & N.F. & 67 & $\mathrm{~F}$ & $\mathrm{G} 2$ & 5 & ++ & - & III & 1.8 & 3.5 & PD & Died \\
\hline 24 & P.L. & 66 & $M$ & G1 & 2 & +++ & ++ & II & 1.6 & 3.0 & SD & PD \\
\hline 25 & R.T. & 48 & $M$ & G1 & 1 & +++ & +++ & I & 0.7 & 4.0 & SD & SD \\
\hline 26 & W.S. & 44 & $\mathrm{~F}$ & G1 & 2 & ++ & - & I & 0.6 & 5.0 & SD & SD \\
\hline 27 & R.T. & 54 & $M$ & G1 & 1 & ++ & ++ & I & 0.1 & 5.5 & SD & SD \\
\hline 28 & A.B. & 57 & $M$ & G1 & 2 & +++ & ++ & I & 1.2 & 6.5 & SD & SD \\
\hline 29 & S.D. & 43 & $\mathrm{~F}$ & G1 & 2 & ++ & - & I & 0.1 & 7.5 & SD & SD \\
\hline 30 & P.R. & 54 & $M$ & G1 & 1 & +++ & ++ & I & 1.0 & 8.0 & SD & SD \\
\hline 31 & K.J & 58 & $\mathrm{~F}$ & G1 & 2 & ++ & + & 1 & 0.5 & 3.5 & SD & SD \\
\hline 32 & F.P & 66 & $M$ & $\mathrm{G} 2$ & 10 & + & - & IV & 2.5 & 3.0 & PD & Died \\
\hline 33 & Z.P. & 59 & $\mathrm{~F}$ & G1 & 2 & +++ & ++ & 1 & 0.3 & 4.0 & SD & SD \\
\hline 34 & J.P & 61 & $\mathrm{~F}$ & G1 & 2 & ++ & + & I & 0.9 & 9.5 & SD & SD \\
\hline 35 & K.P. & 46 & $M$ & G1 & 1 & +++ & ++ & I & 0.1 & 4.5 & SD & SD \\
\hline 36 & D.B. & 67 & $\mathrm{~F}$ & $\mathrm{G} 2$ & 5 & ++ & - & III & 1.7 & 3.5 & PD & Died \\
\hline 37 & G.B. & 55 & $M$ & G1 & 2 & +++ & ++ & 1 & 0.2 & 2.5 & SD & SD \\
\hline 38 & S.T. & 52 & $\mathrm{~F}$ & G1 & 1 & +++ & ++ & I & 1.1 & 10.5 & SD & SD \\
\hline
\end{tabular}

CgA - chromogranin A, Syn - synaptophysin, SD - stable disease, $P D$ - progressive disease. 
5-year survival rate in the patients was $75.3 \%$. The TNM stage is an important prognostic factor [36]. Our study mainly included patients with stage I tumors, reflecting the relatively indolent biological behavior of rectal neuroendocrine tumors, which are characterized by shallow local invasion and few lymph node and distant metastases. In a statistical analysis Brock [37] observed that patients with stage I tumors accounted for $83 \%$ of all patients. Univariate analysis showed that TNM staging was a prognostic factor $(p<0.001)$. The pathological type of tumor also significantly affects the prognosis [38]. According to the WHO 2010 pathological diagnostic criteria for gastrointestinal neuroendocrine tumors based on tissue structures, the degree of differentiation, mitotic rate, and the presence or absence of necrosis, they can be subclassified into three types: G1 neuroendocrine tumors (Ki-67, 0-2\%), G2 neuroendocrine tumors (Ki-67, 2-20\%) and G3 neuroendocrine cancer (Ki-67 over 20\%). The G1 neuroendocrine tumors, which accounted for a large proportion of the rectal neuroendocrine tumors in this study, have a good prognosis. The neuroendocrine carcinomas (G3) account for a smaller proportion of tumors, and they have a significantly worse prognosis, often diagnosed in stage III and IV. In our study patients with $\mathrm{G} 3$ were not included, but 4 patients with $G 2$ were diagnosed as having a stage IV disease at the first patient visit. Other studies have suggested that the diameters of rectal neuroendocrine tumors are closely associated with the invasion depth and with lymph node and distant metastases, and that they have definite prognostic significance [39]. Patients with tumor diameters between 0.1 and $1 \mathrm{~cm}$ have been reported to have a distant metastasis rate of less than $5 \%$ and a 5 -year survival rate of $81 \%$. In contrast, most patients with tumor diameters $\geq 2 \mathrm{~cm}$ had distant metastases and their 5-year survival rate was $18 \%$ to $40 \%$ [40]. In our study, the univariate analysis of prognostic factors showed that tumor diameter was significantly associated with the prognosis $(p=0.001)$. For patients with lesions $0.1-1.9 \mathrm{~cm}$ in diameter, the 5 -year survival rate was $93.3 \%$. In those with tumors $\geq 2 \mathrm{~cm}$ with muscular or serosal invasion, the 5 -year survival rate was $50 \%$, and in those with distant metastasis, the 5-year survival rate was also 50\%. Landry et al. [13] reported that age $\geq 65$ years was a poor prognostic factor for rectal neuroendocrine tumors. In our study, the median age of the patients was 51.7 years, and the survival analysis showed that the prognosis in those over 65 years of age at diagnosis declined. A stratified analysis showed that while the 5-year survival rate of patients under the age of 65 years was $100 \%$, in patients over 65 it was $37.2 \%$. In our study we did not include patients older than
67 years, but according to the Landry project [12] the 5 -year survival rate in those age between 70 and 80 was below $50 \%$. In our study group in the five years of treatment and follow-up (2010-2015) 7 patients (all G2) died; 5 due to progression of disease associated with metastasis to lymph nodes, liver and distant organs and 2 due to systemic diseases. Possible reasons for this might be that patients with rectal neuroendocrine tumors who do not have distant metastases have a better prognosis, and most are able to survive long term. However, elderly patients with underlying diseases usually have decreased organ function and insufficient immune function, and these patients often die due to tumor-specific factors or to their underlying disease. Our studies were conducted on a relatively small study group. Statistical data are significant but conclusions should be extended very carefully. The majority of cases were $\mathrm{G} 1$ neuroendocrine tumors with size up to $1 \mathrm{~cm}$, clinically advanced at stage I. The study group over 65 years of age did not include additional diseases that could be important in the assessment of survival. The inclusion of a larger group of patients with $\mathrm{G} 2$ tumors and size greater than $2 \mathrm{~cm}$ in patients over 65 years of age probably influenced the statistics.

\section{Conflict of interest}

The authors declare no conflict of interest.

\section{References}

1. Modlin IM, Lye KD, Kidd M. A 5-decade analysis of 13,715 carcinoid tumors. Cancer 2003; 97: 934-59.

2. Scherübl H, Streller B, Stabenow R, et al. Clinically detected gastroenteropancreatic neuroendocrine tumors are on the rise: epidemiological changes in Germany. World J Gastroenterol 2013; 19: 9012-9.

3. Fraenkel M, Kim M, Faggiano A, de Herder WW, Valk GD. Incidence of gastroenteropancreatic neuroendocrine tumours: a systematic review of the literature. Endocr Relat Cancer 2014; 21: R153-63.

4. Aytac E, Ozdemir Y, Ozuner G. Long term outcomes of neuroendocrine carcinomas (high-grade neuroendocrine tumors) of the colon, rectum, and anal canal. J Visc Surg 2014; 151: 3-7.

5. Bernick PE, Klimstra DS, Shia J, et al. Neuroendocrine carcinomas of the colon and rectum. Dis Colon Rectum 2004; 47: 163-9.

6. Heo J, Jeon SW, Jung MK, et al. A tailored approach for endoscopic treatment of small rectal neuroendocrine tumor. Surg Endosc 2014; 28: 2931-8.

7. Rindi G, Klöppel G, Couvelard A, et al. TNM staging of midgut and hindgut (neuro) endocrine tumors: a consensus proposal including a grading system. Virchows Arch 2007; 451: 757-62.

8. Solcia E, Sobin LH, Arnold R. Endocrine tumors of the coIon and rectum. In: Pathology and genetics of tumours of the digestive system. Hamilton SR, Aaltonen LA (eds). World Health Organization classification of tumors. IARC Press, Lyon 2010; 137-9. 
9. Modlin IM, Sandor A. An analysis of 8305 cases of carcinoid tumors. Cancer 1997; 79: 813-29.

10. Kos-Kudła B. Treatment of neuroendocrine tumors: new recommendations based on the CLARINET study. Contemp Oncol (Pozn) 2015; 19: 345-9.

11. Kulke MH, Mayer RJ. Carcinoid tumors. N Engl J Med 1999; 340: 858-68.

12. Orloff MJ. Carcinoid tumors of the rectum. Cancer 1971; 28: 175-80.

13. Landry CS, Brock G, Scoggins CR, McMasters KM, Martin RC. A proposed staging system for rectal carcinoid tumors based on an analysis of 4701 patients. Surgery 2008; 144: 460-6.

14. Weinstock B, Ward SC, Harpaz N, Warner RR, Itzkowitz S, Kim MK. Clinical and prognostic features of rectal neuroendocrine tumors. Neuroendocrinology 2013; 98: 180-7.

15. Kim MS, Hur H, Min BS, Baik SH, Lee KY, Kim NK. Clinical outcomes for rectal carcinoid tumors according to a new (AJCC 7th edition) TNM staging system: a single institutional analysis of 122 patients. J Surg Oncol 2013; 107: 835-41.

16. Grycewicz J, Scibór Z, Cwikła JB, Lewiński A, Cypryk K Recurrent hypoglycaemia in a type 2 diabetes patient - diagnostic difficulties. Arch Med Sci 2010; 6: 126-9.

17. Gut P, Czarnywojtek A, Fischbach J, et al. Chromogranin A - unspecific neuroendocrine marker. Clinical utility and potential diagnostic pitfalls. Arch Med Sci 2016; 12: 1-9.

18. Chagpar R, Chiang YJ, Xing Y, et al. Neuroendocrine tumors of the colon and rectum: prognostic relevance and comparative performance of current staging systems. Ann Surg Oncol 2013; 20: 1170-8.

19. Jernman J, Välimäki MJ, Louhimo J, Haglund C, Arola J. The novel WHO 2010 classification for gastrointestinal neuroendocrine tumours correlates well with the metastatic potential of rectal neuroendocrine tumours. Neuroendocrinology 2012; 95: 317-24.

20. Vilor M, Tsutsumi Y, Osamura RY, et al. Small cell neuroendocrine carcinoma of the rectum. Pathol Int 1995 45: 605-9.

21. Naunheim KS, Zeitels J, Kaplan EL, et al. Rectal carcinoid tumors: treatment and prognosis. Surgery 1983; 94: 670-6.

22. Gleeson FC, Levy MJ, Dozois EJ, Larson DW, Wong Kee Song LM, Boardman LA. Endoscopically identified well-differentiated rectal carcinoid tumors: impact of tumor size on the natural history and outcomes. Gastrointest Endosc 2014; 80: 144-51.

23. Kim DH, Lee JH, Cha YJ, et al. Surveillance strategy for rectal neuroendocrine tumors according to recurrence risk stratification. Dig Dis Sci 2014; 59: 850-6.

24. Blicharz-Dorniak J, Kos-Kudła B, Kudła M, Foltyn W. Polypeptide growth factors in gastroenteropancreatic neuroendocrine tumours. Endokrynol Pol 2007; 58: 42-9.

25. Kim GU, Kim KJ, Hong SM, et al. Clinical outcomes of rectal neuroendocrine tumors $\leq 10 \mathrm{~mm}$ following endoscopic resection. Endoscopy 2013; 45: 1018-23.

26. Jeon JH, Cheung DY, Lee SJ, et al. Endoscopic resection yields reliable outcomes for small rectal neuroendocrine tumors. Dig Endosc 2014; 26: 556-63.

27. Kunikowska J, Królicki L, Sowa-Staszczak A, Pawlak D, Hubalewska-Dydejczyk A, Mikołajczak R. Nephrotoxicity after PRRT - still a serious clinical problem? Renal toxicity after peptide receptor radionuclide therapy with 90Y-DOTATATE and 90Y/177Lu-DOTATATE. Endokrynol Pol 2013; 64: 13-20.

28. Zhou FR, Huang LY, Wu CR. Endoscopic mucosal resection for rectal carcinoids under micro-probe ultrasound guidance. World J Gastroenterol 2013; 19: 2555-9.
29. Kos-Kudła B, Bolanowski M, Handkiewicz-Junak D, Jarząb B. Diagnostic and therapeutic guidelines for gastrointestinal neuroendocrine tumors (recommended by the Polish Network of Neuroendocrine Tumors). Endokrynol Pol 2008; 59: 41-56.

30. Foltyn W, Zajęcki W, Marek B. The value of the Ki-67 proliferation marker as a prognostic factor in gastroenteropancreatic neuroendocrine tumours. Endokrynol Pol 2012; 63: 362-6.

31. Kunikowska J, Królicki L, Sowa-Staszczak A, et al. Polish experience in peptide receptor radionuclide therapy. Recent Results Cancer Res 2013; 194: 467-78.

32. Pach D, Sowa-Staszczak A, Kunikowska J, et al. Repeated cycles of peptide receptor radionuclide therapy (PRRT) results and side-effects of the radioisotope 90Y-DOTA TATE, 177Lu-DOTA TATE or 90Y/177Lu-DOTA TATE therapy in patients with disseminated NET. Radiother Onco 2012; 102: 45-50.

33. Kunikowska J, Królicki L, Hubalewska-Dydejczyk A, Mikołajczak R, Sowa-Staszczak A, Pawlak D. Clinical results of radionuclide therapy of neuroendocrine tumours with 90Y-DOTATATE and tandem 90Y/177Lu-DOTATATE: which is a better therapy option?. Eur J Nucl Med Mol Imaging 2011; 38: 1788-97.

34. Akahoshi K, Motomura Y, Kubokawa M, et al. Endoscopic submucosal dissection of a rectal carcinoid tumor using grasping type scissors forceps. World J Gastroenterol 2009; 15: 2162-5.

35. Abe T, Kakemura T, Fujinuma S, Maetani I. Successful outcomes of EMR-L with 3D-EUS for rectal carcinoids compared with historical controls. World J Gastroenterol 2008; 14: 4054-8.

36. Kasuga A, Chino A, Uragami N, et al. Treatment strategy for rectal carcinoids: a clinicopathological analysis of 229 cases at a single cancer institution. J Gastroenterol Hepatol 2012; 27: 1801-7.

37. Gut P, Fischbach J, Kamiński G, Ruchała M. Contemporary methods of therapy and follow-up of neuroendocrine tumours of the gastrointestinal tract and the pancreas. Contemp Oncol (Pozn) 2012; 16: 371-5.

38. Fischbach J, Gut P, Matysiak-Grześ M, et al. Combined octreotide and peptide receptor radionuclide therapy ((90)Y-DOTA-TATE) in case of malignant insulinoma. Neuro Endocrinol Lett 2012; 33: 273-8.

39. Jetmore AB, Ray JE, Gathright JB, McMullen KM, Hicks TC, Timmcke AE. Rectal carcinoids: the most frequent carcinoid tumor. Dis Colon Rectum 1992; 35: 717-25.

40. Starzyńska T, Deptała A, Królicki L, et al; Consensus Conference; Polish Network of Neuroendocrine Tumours. Colorectal neuroendocrine neoplasms - management guidelines (recommended by the Polish Network of Neuroendocrine Tumours). Endokrynol Pol 2013; 64: 494-504. 